\title{
Modelling vapour transport in Surtseyan bombs
}

\author{
Mark J. McGuinness ${ }^{\mathrm{a}, \mathrm{b}, *}$, Emma Greenbank $^{\mathrm{a}}$, C. Ian Schipper ${ }^{\mathrm{c}}$ \\ a School of Mathematics and Statistics, Victoria University of Wellington, New Zealand \\ b MACSI, University of Limerick, Ireland \\ c School of Geography, Environment, and Earth Sciences, Victoria University of Wellington, New Zealand
}

\section{A R T I C L E I N F O}

\section{Article history:}

Received 26 November 2015

Revised 21 March 2016

Accepted 22 March 2016

Available online $\mathrm{xxxx}$

\section{Keywords:}

Surtseyan bombs

Mathematical model

Pressure transients

Fragmentation

Steam escape times

\begin{abstract}
A B S T R A C T
We address questions that arise if a slurry containing liquid water is enclosed in a ball of hot viscous vesicular magma ejected as a bomb in the context of a Surtseyan eruption. We derive a mathematical model for transient changes in temperature and pressure due to flashing of liquid water to vapour inside the bomb. The magnitude of the transient pressure changes that are typically generated are calculated together with their dependence on material properties. A single criterion to determine whether the bomb will fragment as a result of the pressure changes is derived. Timescales for ejection of water vapour from a bomb that remains intact are also revealed.
\end{abstract}

(c) 2016 Elsevier B.V. All rights reserved.

\section{Introduction}

A driving purpose in volcanology is to better understand observations of active volcanoes and of the deposits that result from eruptions. This is particularly so in the emergent field of Surtseyan eruptions, characterised by their unique bulk interactions between molten magma and large quantities of water. This paper aims to inform the ongoing discussion of key mechanisms of Surtseyan eruptions by developing a transient mathematical model of heat and mass transport inside a Sursteyan bomb, assuming that a wet slurry inclusion has been encapsulated by a body of hot vesicular magma at the instant of ejection (Kokelaar, 1983; Schipper \& White).

Surtseyan eruptions take their name from their resemblance to the eruptions that formed the new island of Surtsey off the coast of Iceland in 1963 (Kokelaar, 1983; Thorarinsson, 1965; Thorarinsson, 1967a; Thorarinsson, 1967b; Thorarinsson, 1968; Thorarinsson et al., 1964). Defined as shallow subaqueous explosive basaltic volcanic eruptions (White and Houghton, 2000), Surtseyan eruptions are violent and are characterised by the ejection of silent tephra jets, with bombs shooting out of each jet, trailing black comet-like tails that turn white as steam condenses (Thorarinsson, 1967b). Large bodies of water with ready access to the vent surface, mix with ejected tephra that has fallen or slipped back into the top of the vent, to form a slurry that readily penetrates molten vesicular magma (Kokelaar, 1983; Kokelaar, 1986). Textural studies (Murtagh and White, 2013; Schipper \& White) provide evidence in tephra of intact bombs, highly vesicular and highly

\footnotetext{
* Corresponding author.

E-mail address: Mark.McGuinness@vuw.ac.nz (M.J. McGuinness).
}

permeable, each containing a number of inclusions. Each inclusion consists of material similar to that in the parent bomb, surrounded by a void space. Murtagh and White (Murtagh and White, 2013) note that "many lapilli also contain previously formed pyroclasts as inclusions", and noted evidence that "erupting magma entrained previously formed pyroclasts". One explanation for this morphology would be that the void space is associated with water that has vented during the ejection process, leaving the bomb intact. Steam venting during ejection is also evidenced by the vapour trails observed behind bombs during ejection (Thorarinsson, 1967b; Schipper \& White).

The term bulk interaction steam explosivity, referring to water encapsulated by hot magma and prevented from escaping, is the third of the processes listed by Kokelaar (Kokelaar, 1986), for forming clasts in a basaltic volcano, that is, for rupturing magma. Kokelaar also argues that this process is a key ejection mechanism in Surtseyan eruptions, causing "a violent and continuous expansion that is manifested as a jet of tephra". Murtagh and White (Murtagh and White, 2013) also note in their conclusions the important role played by magma-water explosivity in driving fragmentation throughout the Surtseyan eruption at Black Point volcano in California. So it is perhaps surprising to consider, alongside of this violent explosivity associated with water-magma interactions, the possibility that there is also a nonviolent interaction between ejected vesicular bombs and enclosed slurry, evidenced by observations of steam trails and voids.

Given the observations noted above and in (Schipper \& White), our interest in this paper is focussed on the interaction between bulk water enclosed as a slurry and the ejected magma bomb containing it, and on the possibility that, and the conditions under which, the bomb does not rupture. We ignore the effects of distributed water coming out of 
solution due to pressure decrease as magma rises, except to model the bomb as a porous medium, vesiculated by that process. We seek a criterion for bomb rupture, and timescales for steam escape.

\subsection{Model motivation}

A calculation of the pressure increase consequent on instantaneously heating a small inclusion of liquid water from say $370 \mathrm{~K}$ at one atmosphere to $1200 \mathrm{~K}$ at pressure $P_{2}$, can be made by using the ideal gas equation $P V=n R T$. Note that for instantaneous heating, the volume $V$ and the number of moles $n$ of water is the same before and after heating the water. $T$ is the absolute temperature, and $P$ is the pressure in Pa.

Since the number of moles of water in an inclusion of effective volume $V$ is given by $n=\rho_{l} V / M$, where $\rho_{l}$ is liquid density and $M$ is the mass of one mole of water, vaporising all of the liquid water affects pressure in two ways - the density ratio of liquid to vapour phase of water gives a higher number of moles than starting with only vapour, by a factor of about $1000 / 0.6 \approx 1700$, and the temperature increase of the vapour phase from $370 \mathrm{~K}$ to $1200 \mathrm{~K}$ raises the pressure by a factor of about three:

$\frac{n R}{V}=$ constant $=\frac{P}{T} \approx \frac{1700 \times 10^{5}}{370}=\frac{P_{2}}{1200}$.

The combination of increased number of moles and increased temperature, starting at one atmosphere, gives $P_{2} \approx 550 \mathrm{MPa}$.

Tensile strengths of small rock samples range up to $66 \mathrm{MPa}$ for basalt (Goodman, 1989), p.83, and for vesicular basalt will be considerably less than this value, with shock tube experiments (Koyaguchi et al., 2008) indicating rupture at pressure differences ranging from 2-30 MPa for hot $\left(850^{\circ} \mathrm{C}\right)$ and cold vesicular magmas with porosities ranging from $0.05-0.8$. Most of the samples with porosities above 0.2 ruptured at less than 15 MPa. Koyaguchi et al. (Koyaguchi et al., 2008) calculate an effective tensile strength of 2.18 MPa for their samples from Unzen and Monserrat, with an error of about $\pm 2 \mathrm{MPa}$, after correcting for the effect of porosity.

Hence, instantaneous heating of an inclusion leads to pressure differences that far exceed the expected tensile strength of the bomb containing them, yet intact bombs are routinely encountered. That is, if water is encapsulated by hot magma and is prevented from escaping as steam, simple calculations indicate the magma will be ruptured. However, heating and flashing to steam of enclosed liquid is not instantaneous, and enclosed high-pressure vapour can escape a vesicular bomb since it is effectively a porous medium. There is then a race between the heating of liquid in an inclusion, and the escape of the vapour generated through the surrounding porous medium, that determines how large a pressure difference is created. The pressures generated would be expected to depend on the thermal and transport properties of the magma bomb, and this dependence is of interest, leading perhaps to a criterion for fragmentation of the bomb (Spieler et al., 2004; Mueller et al., 2008), and a timescale for escape of the water if the bomb does not fragment. These considerations provide the motivation for developing a mathematical model that gives the time-dependent pressure behaviour when a liquid water inclusion is heated by a bomb.

\section{Mathematical model}

We model the transient heating of a single inclusion placed at the centre of a spherical ball of hot vesicular magma at the instant of ejection. The magma and inclusion are treated as porous media. The inclusion is taken to be a sphere of radius $R_{1}$, and the magma to have radius $R_{2}$, as illustrated in Fig. 1. SI units are used throughout unless stated. Observations suggest $R_{2}$ values range from several millimetres to more than a metre. We will use $10 \mathrm{~cm}$ here for illustration purposes.

We consider the inclusion to be a slurry of ash and lapilli and water, and that the water can escape the ball only by flowing as a vapour

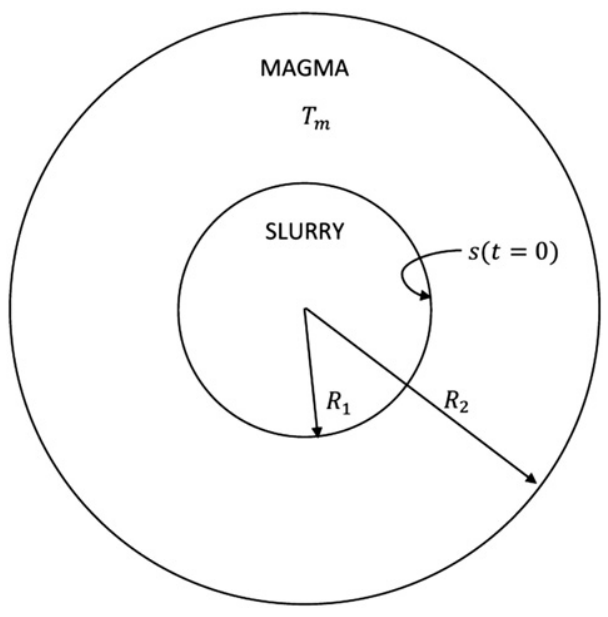

Fig. 1. A sketch of the model of a spherical Surtseyan bomb with a spherical slurry inclusion placed at its centre. The inclusion has radius $R_{1}$. The bomb has initial temperature $T_{m}$ and radius $R_{2}$. Not to scale.

through the porous magma, but that the solid component of the slurry remains in place, as observed in intact bombs. Initially the included water is assumed to be liquid at or near boiling point and at atmospheric pressure. The only other water vapour present in our model is that in the vesicles in the surrounding magma, which has come out of solution as the rising magma cooled. The magma ball is flying through the air at the head of a plume in a tephra jet, with its surface effectively at atmospheric pressure but very hot.

We model the heating of the inclusion by conduction from the surrounding hot magma, and we model the consequent flash to steam to obtain the pressure transients as steam is generated at the inclusion and then flows out through the porous magma due to the pressure differences generated by heating. For simplicity we separate the heating and flowing problems.

\subsection{Temperature model}

The radius of the slurry inclusion is assumed to be small enough that the initial temperature $T_{m}$ of the surrounding magma does not change significantly during the time that it is heating the enclosed water and solids. A temperature gradient between the surface of the inclusion at a time-varying temperature $T$ and the hot magma is estimated by a heat balance approach, so that the rate of heat flow into the inclusion from the magma is

$4 \pi K R_{1}^{2} \frac{\partial T_{\text {magma }}}{\partial r}$

where $K$ is the thermal conductivity of the hot magma, and $T_{\text {magma }}$ is the actual temperature of the surrounding magma, which varies with radial distance $r$ and time $t$. We approximate the temperature gradient in the magma by $\left(T_{m}-T\right) / R_{T}$ where $R_{T} \approx 0.3 R_{1}$. The thermal lengthscale $R_{T}$ is calculated in the Appendix $A$, and it depends on both sensible and latent heat changes.

Then the rate of heat flow into the inclusion is estimated as

$4 \pi K R_{1}^{2}\left(\frac{T_{m}-T}{0.3 R_{1}}\right)$

The spatial origin is taken to be at the centre of the inclusion, and time is zero at the instant of entrainment. We match the rate of change of internal energy of the inclusion to the rate of heat flow into it,

$\frac{4}{3} \pi R_{1}^{3} \rho c_{p} \frac{d T}{d t}=4 \pi K R_{1}^{2}\left(\frac{T_{m}-T}{0.3 R_{1}}\right)$ 
where $\rho=\phi_{1} \rho_{l}+\left(1-\phi_{1}\right) \rho_{m}$ is the effective density of the inclusion, $\phi_{1}$ is the porosity of the inclusion, $\rho_{l}$ is the density of liquid water, $\rho_{m}$ is the density of solid magma, and $c_{p}$ is the effective heat capacity of the inclusion at constant pressure (see the Appendix A for more details). This effective heat capacity varies with temperature, and jumps at the boiling point of the liquid due to the specific heat of vaporisation, but we take a constant effective value in order to simplify the thermal problem sufficiently to solve it separately from the pressure problem.

The temperature at the surface of the inclusion must then satisfy the equation

$\frac{d T}{d t}=\frac{10 K}{\rho c_{p} R_{1}^{2}}\left(T_{m}-T\right)$

which has the solution

$T=T_{m}-\left(T_{m}-T_{0}\right) e^{-\alpha \mathrm{t}}$

where $T_{0}$ is the initial temperature of the inclusion upon entrainment, and

$\alpha=\frac{10 K}{\rho c_{p} R_{1}^{2}}$

So our simple model has the temperature at the surface of the inclusion increasing and approaching the magma temperature, with a timescale of $1 / \alpha$.

\subsection{Pressure model}

Conservation of vapour mass in the surrounding hot magma can be expressed as

$\frac{\partial\left(\phi_{2} \rho_{v}\right)}{\partial t}=-\nabla \cdot\left(\mathbf{v} \phi_{2} \rho_{v}\right)$

where $\rho_{v}$ is the density of vapour, $\phi_{2}$ is the porosity of the magma, and $\mathbf{v}$ is the fluid velocity (bold font indicating a vector quantity) in the pores. This may be combined with Darcy's law for the volume flux q (volume per unit area per second) of a fluid through a porous medium,

$\phi_{2} \mathbf{v}=\mathbf{q}=-\frac{k}{\mu_{v}} \nabla p$,

where $k$ is the permeability of the porous magma, $p$ is the pressure of the water vapour, and $\mu_{v}$ is the dynamic viscosity of the water vapour. Permeability and dynamic viscosity are taken to be constant here. Then we have

$\frac{\partial\left(\phi_{2} \rho_{v}\right)}{\partial t}=\nabla \cdot\left(\frac{k \rho_{v}}{\mu_{v}} \nabla p\right)$

The ideal gas law relates vapour density to vapour pressure:

$\rho_{v}=\frac{p M}{R T_{\text {magma }}}$

where $R=8.314 \mathrm{~J} \mathrm{~K}^{-1} \mathrm{~mol}^{-1}$ is the universal gas constant, and $M$ is the molar mass of water $\left(\mathrm{kg} \mathrm{mol}^{-1}\right)$. Equilibrium between vapour temperature and magma temperature is assumed. We again neglect changes in magma temperature, setting $T_{\text {magma }} \approx T_{m}$. Then using the ideal gas law to replace vapour density in Eq. (1) gives a nonlinear diffusion equation for vapour pressure,

$\frac{\partial p}{\partial t}=\left(\frac{k}{\phi_{2} \mu_{v}}\right) \nabla \cdot(p \nabla p)$
The initial condition is taken to be that $p(r, 0)=0$, that is, that the partial pressure of water vapour in the vesicular magma is negligible. The total pressure at the surface of the bomb may be assumed to be one atmosphere. The partial pressure of water vapour there can be up to one atmosphere, much smaller than critical fragmentation pressures. Similarly, the boundary condition at the surface of the hot magma at $r=$ $R_{2}$ is assumed to also correspond to negligible partial pressure of water vapour,

$p=0, r=R_{2}$.

The other boundary condition to be applied is at the flashing front in the inclusion. This is considered in the following subsection.

\subsection{Flashing front}

The flow of heat to the inclusion vaporises the water there, providing a source of vapour that flows into the surrounding magma. We model this source as a flashing front that propagates into the inclusion at a rate governed by a heat balance between the heat conducted to the inclusion from the surrounding magma, and the latent heat required to move the flashing front some distance into the inclusion. This is a classic Stefan problem for propagation of a change of phase.

The amount of heat provided to the flashing front located at radius $s(t)$ in time $\Delta t$ by conduction from the hot magma is

$4 \pi s^{2} K \frac{d T}{d r} \Delta t$

We approximate $d T / d r$ by $\left(T_{m}-T\right) / R_{T}$ and use our temperature solution to obtain the following expression for the amount of conductive heat provided:

$4 \pi s^{2} K\left(T_{m}-T_{0}\right) \frac{e^{-\alpha \mathrm{t}}}{R_{T}} \Delta t$.

We neglect changes in sensible heat and heat loss due to vapour flowing outwards from the flashing front. We assume that in time $\Delta t$ the flashing front advances a distance $\Delta s$ towards the origin. We match the conductive heat provided in time $\Delta t$ with the heat required to vaporise the water in a spherical shell of thickness $\Delta s$,

$4 \pi s^{2} \Delta s \rho_{l} \phi_{1} h_{v l}$

where $h_{\mathrm{vl}} \mathrm{J} \mathrm{kg}^{-1}$ is the specific heat of vaporisation of water. Then, equating heat supplied to heat required, taking the limit as $\Delta t$ (and hence $\Delta s$ ) approaches zero, and noting that $\dot{s}=\lim _{\Delta t \rightarrow 0} \Delta s / \Delta t$, we obtain an expression for the inwards speed of the flashing front,

$|\dot{S}|=\frac{K\left(T_{m}-T_{0}\right) e^{-\alpha t}}{0.3 \rho_{l} \phi_{1} h_{v l} R_{1}}$.

Starting with $s=R_{1}$ at $t=0$, this implies that

$s(t)=B\left(e^{-\alpha t}-1\right)+R_{1}$

where $B / R_{1}$ is a Stefan number, and

$B=\frac{\left(T_{m}-T_{0}\right) \rho c_{p} R_{1}}{3 \rho_{l} \phi_{1} h_{\mathrm{vl}}}$.

Hence flashing of liquid in the inclusion is completed when $s=0$, that is, after $t_{0}$ seconds, where

$t_{0}=-\frac{1}{\alpha} \ln \left(1-\frac{R_{1}}{B}\right)$. 
Note that for $t_{0}$ to be a real number requires that the Stefan number $B / R_{1}$ be greater than one, that is, that the change in sensible heat at the inclusion exceeds the latent heat required to vaporise all of the liquid in it. If for example the temperature of the surrounding magma is insufficient to completely vaporise the liquid in the inclusion, then $t_{0}$ has no real value since the flashing front never reaches the origin $(s=0)$.

\subsubsection{Inner boundary condition}

The vapour pressure gradient in the magma at the flashing front resulting from the generation of steam in the inclusion is then obtained by noting that the vapour generated must all flow outwards into the porous magma surrounding the inclusion. The mass of vapour generated per second over the entire flashing front is

$-\phi_{1} \rho_{l} 4 \pi s^{2} \dot{s}$

and the total mass flow rate of vapour that flows away from the flashing front into the magma is

$-4 \pi s^{2} \frac{k \rho_{v}}{\mu_{v}} \nabla p$

Equating these and applying the ideal gas law gives

$\left(\frac{k}{\phi_{2} \mu_{v}}\right) p \nabla p=\frac{R T_{m} \phi_{1} \rho_{l} \dot{s}}{M \phi_{2}}$

This provides a flux boundary condition at the inner boundary $r=$ $s(t)$ between the origin and $R_{1}$, until $t=t_{0}$ and there is no more liquid left in the inclusion. After time $t_{0}$, the boundary condition at the origin is then

$\left(\frac{k}{\phi_{2} \mu_{v}}\right) p \nabla p=0$

To summarise, the dimensional problem to solve is

$\frac{\partial p}{\partial t}=D \frac{1}{r^{2}} \frac{\partial}{\partial r}\left(p r^{2} \frac{\partial p}{\partial r}\right)$

$p=0, r=R_{2}$

$D p \frac{\partial p}{\partial r}=\left\{\begin{array}{cl}\frac{R T_{m} \phi_{1} \rho_{l} \dot{s}}{M \phi_{2}}, & t<t_{0} \\ 0, & t \geq t_{0}\end{array}, r=s(t)\right.$

$\dot{s}=-\frac{K\left(T_{m}-T_{0}\right) e^{-\alpha \mathrm{t}}}{0.3 \rho_{l} \phi_{1} h_{\mathrm{vl}} R_{1}}$

$\alpha=\frac{10 K}{\rho c_{p} R_{1}^{2}}$

$D=\frac{k}{\phi_{2} \mu_{v}}$

$t_{0}=-\frac{1}{\alpha} \ln \left(1-\frac{R_{1}}{B}\right)$

$B=\frac{\left(T_{m}-T_{0}\right) \rho c_{p} R_{1}}{3 \rho_{l} \phi_{1} h_{\mathrm{vl}}}$

and the flashing front at $r=S(t)$ begins at $r=R_{1} \ll R_{2}$ and reaches zero in time $t_{0}$. $D p$ is the nonlinear diffusivity in this nonlinear Stefan diffusion problem for vapour pressure, which has a moving boundary at the flashing front in the inclusion.

We now rescale and non-dimensionalize the problem, in order to identify key parameters and timescales.

\section{Non-dimensional model}

The model Eqs. (4) to (11) are rescaled and non-dimensionalised by scaling pressure on the tensile strength $p_{c}$ of vesicular magma, scaling time on the time $t_{0}$ required to flash all of the included liquid to vapour, and scaling $r$ and $s$ on the radius $R_{2}$ :

$\tilde{p}=\frac{p}{p_{c}}, \tilde{t}=\frac{t}{t_{0}}, \tilde{r}=\frac{r}{R_{2}}, \tilde{s}=\frac{s}{R_{2}}, \tilde{D}=\frac{t_{0} p_{c} D}{R_{2}^{2}}$.

Note that typically the Fourier number $\alpha t_{0} \approx 0.04$, which is small enough that we can approximate $e^{-\alpha t_{0} t} \approx 1$ for $t<1$, and the nondimensional flash front speed then becomes constant, $\dot{s}=-\frac{R_{1}}{R_{2}}$. We now have a simplified timescale for flash completion

$t_{0}=\frac{0.3 \phi_{1} R_{1}^{2} \rho_{l} h_{\mathrm{vl}}}{K\left(T_{m}-T_{0}\right)}$.

The tildes are dropped, to obtain

$\frac{\partial p}{\partial t}=\frac{D}{r^{2}} \frac{\partial}{\partial r}\left(p r^{2} \frac{\partial p}{\partial r}\right)$

$p(r, 0)=0, p(1, t)=0$

$D p \frac{\partial p}{\partial r}=\left\{\begin{array}{cc}-E, & t<1 \\ 0, & t \geq 1\end{array}, r=s(t)\right.$

$s(t)=\left\{\begin{array}{cc}\frac{R_{1}}{R_{2}}(1-t), & t<1 \\ 0, & t \geq 1\end{array}\right.$

$E=\frac{R_{1} R T_{m} \phi_{1} \rho_{l}}{R_{2} M \phi_{2} p_{c}}$

$D=\frac{0.3 p_{c} h_{v \mathrm{l}} \rho_{l} \phi_{1} k R_{1}^{2}}{K \mu_{v}\left(T_{m}-T_{0}\right) \phi_{2} R_{2}^{2}}$.

Our model is a nonlinear diffusion equation for $p$ with diffusivity $D p$ and a constant flux $E$ at the flashing surface which is the moving boundary $s(t)$. This flux turns off at $t=1$. The total mass flow rate of vapour out of the inclusion reduces as $s^{2}$ reduces in time, that is, like a quadratic in time, until it reaches zero at $t=1$. The critical value of nondimensional pressure at which bomb rupture is anticipated is if $p$ reaches the value one somewhere inside.

The nondimensional model Eqs. (12)-(15) now depend on three parameters, $D, E$, and $R_{1} / R_{2}$.

Note that as $R_{1}$ increases, the flux $E$ increases near the origin. But dimensionless diffusivity $D$ also increases as $R_{1}$ increases, so it is difficult to deduce pressure behaviour at the origin directly from these parameters. In the next section, numerical solutions guide us to further helpful simplifications.

\subsection{Numerical solutions - fixed flash front}

We use Matlab's pdepe command to solve Eqs. (12)-(15). As a first step, we fix the value of dimensionless $s=\varepsilon \equiv R_{1} / R_{2}$, that is, we take a constant flash position at the surface of the inclusion for the flash front, modelling the inclusion as delivering vapour to that fixed front for dimensionless time $t_{0}$. This delivers a larger total amount of vapour than in the actual model, since it does not take account of the reducing total area of the flashing surface, and pressure values obtained at this fixed value of $s$ then provide an upper limit on the actual pressure values for $s(t) \rightarrow 0$. This upper limit turns out to be very useful.

Typical values for constants and parameters used in numerical solutions unless noted otherwise are listed in Tables 1 and 2. Initial values 
Table 1

Physical constants used.

\begin{tabular}{lll}
\hline Constant & Value & Units \\
\hline$p_{c}$ & $2 \times 10^{6}$ & $\mathrm{~Pa}$ \\
$h_{v l}$ & $2 \times 10^{6}$ & $\mathrm{~J} \mathrm{~kg}^{-1}$ \\
$\rho_{l}$ & 1000 & $\mathrm{~kg} \mathrm{~m}^{-3}$ \\
$k$ & $10^{-14}$ & $\mathrm{~m}^{2}$ \\
$K$ & 2 & $\mathrm{~W} \mathrm{~m}^{-1} \mathrm{~K}^{-1}$ \\
$\mu_{v}$ & $3 \times 10^{-5}$ & $\mathrm{~Pa} \mathrm{~s}^{-1}$ \\
$M$ & $18 \times 10^{-3}$ & $\mathrm{~kg} \mathrm{~mol}^{-1}$ \\
$R$ & 8.314 & $\mathrm{~J} \mathrm{~K}^{-1} \mathrm{~mol}^{-1}$ \\
$T_{m}$ & 1300 & $\mathrm{~K}$ \\
$T_{0}$ & 300 & $\mathrm{~K}$ \\
$\phi_{2}$ & 0.4 & \\
$\phi_{1}$ & 0.4 & $\mathrm{~m}$ \\
$R_{1}$ & 0.001 & $\mathrm{~m}$ \\
$R_{2}$ & 0.1 & \\
\hline
\end{tabular}

for pressure $p$, and the boundary value at $r=1$, are all taken to be $1.0 \times 10^{5} \mathrm{~Pa}$ in numerical simulations, rather than the zero value described above. This only affects later computations of steam release curves.

The numerical solutions reveal an interesting feature - the pressure at the fixed surface of the inclusion quickly equilibrates, as illustrated in Fig. 2. This rapid equilibration is observed over a wide range of parameters, independently of whether pressure changes are visible in graphs at $r=1$ when $t=1$, or not.

The timescale for pressure to reach a steady value at the surface of the inclusion is readily obtained, in a manner analogous to the exact solution in the book by Crank (Crank, 1974), p.32. There an exact solution proportional to $\operatorname{erfc}(\eta)$ is given for a linear diffusion equation with a point source of constant flux at the origin, in an infinite medium, which depends on the well-known similarity variable $\eta=r /(2 \sqrt{D t})$. That solution equilibrates when $\eta \approx 1$, giving a timescale $r^{2} /(4 D)$.

For our nonlinear problem, we can similarly take the source of vapour to be at the origin. In fact it is not possible to specify a boundary value or flux at the origin in spherical coordinates. What is imposed is the correct total flux at some radius $\varepsilon$, which is taken to be arbitrarily close to the origin. We are interested in early times when the solution may be approximated by a solution on an infinite domain. The lengthscale for the point of interest is $r=\varepsilon$.

Our model may be compared to a linear one by rewriting the diffusion Eq. (12), by multiplying both sides by $2 p$ and rearranging to obtain the form

$\frac{\partial p^{2}}{\partial t}=\frac{D p}{r^{2}} \frac{\partial}{\partial r}\left(r^{2} \frac{\partial p^{2}}{\partial r}\right)$

which is linear in the variable $p^{2}$, provided that one uses an average pressure value $\bar{p}$ in the diffusivity term $D p$. This process is well-known in geothermal reservoir literature, e.g. (Moench, 1979; Grant, 1978; Moench and Atkinson, 1977), where taking an average pressure is a good approximation.

The timescale $t_{s}$ for pressure changes at early times is then proportional to the ratio of lengthscale squared to diffusivity $D \bar{p}$,

$t_{s}=\frac{\varepsilon^{2}}{4 D \bar{p}}$

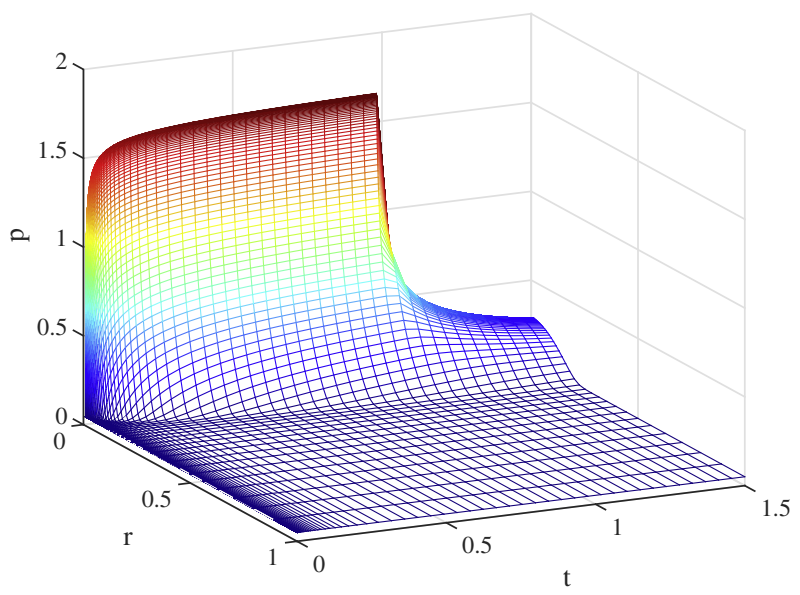

Fig. 2. Numerical solutions to the nondimensional pressure equations, showing pressure versus radius and time (all nondimensional). The flashing front is approximated by fixing it at the initial radius of the inclusion. Parameters are as listed in Table 1.

where $\bar{p}$ is some average value of pressure, of order one for pressures of interest. Hence the condition for the pressure to equilibrate at the fixed flash front before running out of steam is $t_{s}<1$, that is, $\varepsilon^{2}<4 D \bar{p}$. Values for $D$ vary, but a typical small value for typical parameter values is 0.02 , giving $\varepsilon=R_{1} / R_{2}<0.3$. Most values of $R_{1}$ that we consider are well below this value $\left(0.3 R_{2}\right)$, since we are assuming little thermal impact on the surrounding magma due to flashing the inclusion, implying that the timescale for pressure to equilibrate at the flashing front is typically much less than the time for the inclusion to boil dry.

\subsection{Numerical results - moving flash front}

The moving boundary problem may be solved numerically by following the flashing front. We could do this by solving the fixed-front problem for a very short time, then updating the front position, remeshing, and interpolating the previous solution to provide new initial values for the next step. Note that an alternative approach is to specify the correct (reducing with time) total flux $-4 \pi E s^{2}(t)$ at some small fixed value of $\varepsilon$, rather than the constant flux $-E$ at a variable flash front location. Then pressure values at radii less that $s(t)$ are ignored, and the maximum pressure is found by interpolating the pressures obtained to evaluate at $r=s(t)$. The second approach, which corresponds to the point source method for diffusion problems in porous media, is much faster, since it does not require re-meshing and interpolating. Both methods have been compared and found to give the same results on $[s(t), 1]$.

The results are illustrated in Fig. 3. The most noticeable effect is that the stable maximum pressure seen in the fixed boundary simulations is almost reached before the pressure at the flashing front reduces due to its movement towards the origin. This reduces the maximum value only slightly from that in the fixed front simulations.

In both of these simulations, the maximum pressure computed numerically is observed to rise above one, which should rupture the surrounding rock, assuming it has an effective tensile strength of $2 \mathrm{MPa}$. Increasing the permeability, as in Fig. 4, is one way to reduce the nondimensional maximum pressure so that it never rises above the critical value of one. Increasing the radius of the inclusion from $1 \mathrm{~mm}$ to $1 \mathrm{~cm}$, on the other hand, has a relatively small effect on the maximum pressure, as may be observed by comparing Fig. 5 with Fig. 3 .

\section{Steady-state solutions}

The rapid stabilisation of pressure at the surface of the inclusion means that the steady-state solution for pressure in the case of a 


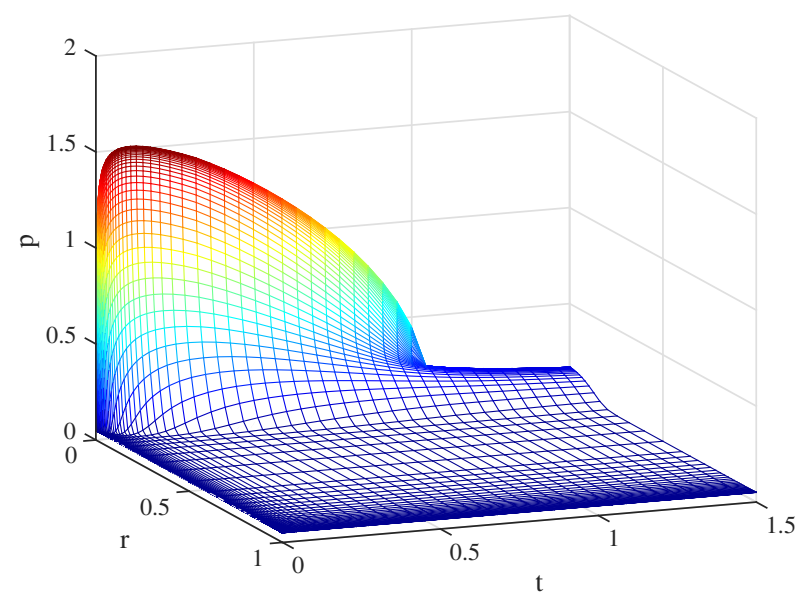

Fig. 3. Numerical solutions to the nondimensional pressure equations, showing pressure versus radius and time (all nondimensional), when the flashing front is allowed to travel towards the origin. The radial mesh, initial conditions, and the flashing front location $s(t)$ are tracked. Parameters are as listed in Table 1.

constant and persistent source of vapour at $r=\varepsilon$ may be used to provide an approximation to the maximum value of pressure achieved there, even when the source reduces with time and does turn off at $t=1$. A steady state everywhere is not typically achieved by the time $t=1$, as is evident in Fig. 2, but the value of pressure at $r=\varepsilon$ predicted by the steady-state solution will provide a fairly close upper bound to the actual pressure there, since it reaches a stable value relatively quickly.

The steady-state solution satisfies

$\frac{D}{r^{2}} \frac{\partial}{\partial r}\left(p r^{2} \frac{\partial p}{\partial r}\right)=0$

which implies that

$r^{2} \frac{\partial p^{2}}{\partial r}=c_{1}$

where $c_{1}$ is a constant of integration. The steady solution is then

$p^{2}=-\frac{c_{1}}{r}+c_{2}$

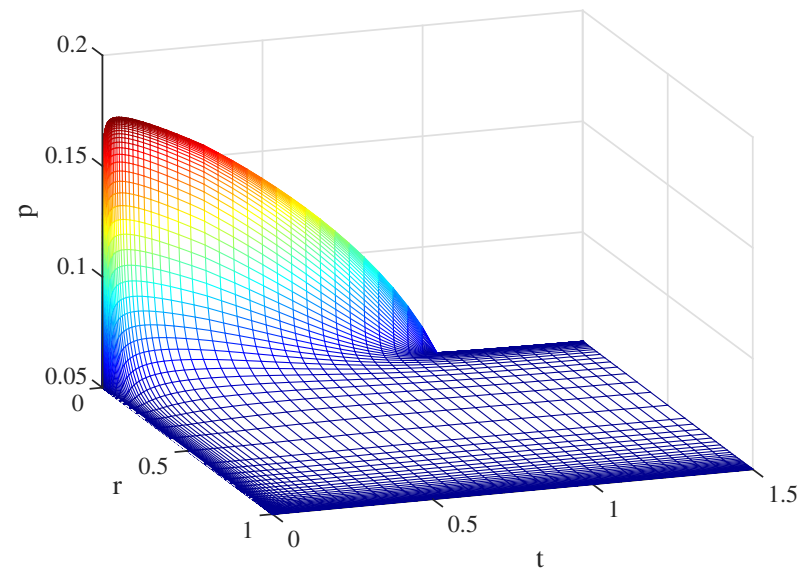

Fig. 4. Numerical solutions to the nondimensional pressure equations, showing pressure versus radius and time (all nondimensional). The inner boundary moves with the flashing front, as in the previous figure. The permeability is $10^{-12} \mathrm{~m}^{2}$. Other parameters are as in Table 1.

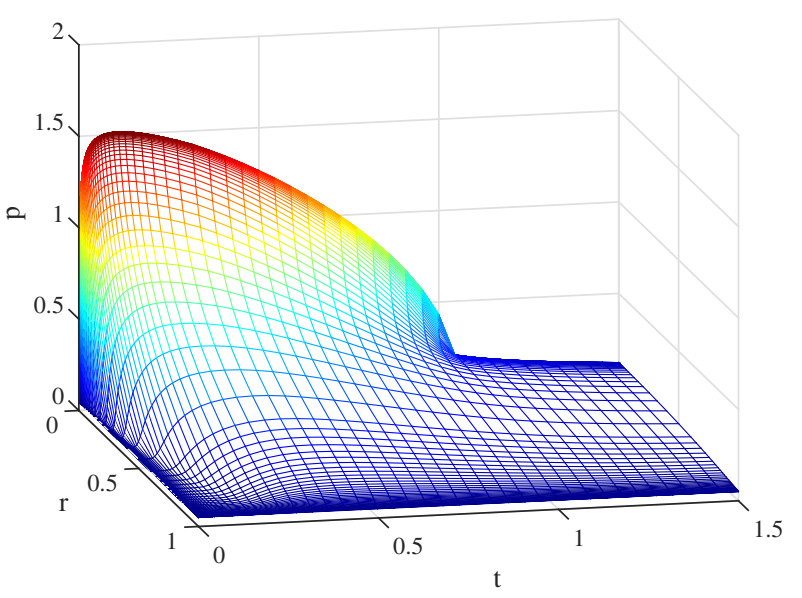

Fig. 5. Numerical solutions to the nondimensional pressure equations, showing pressure versus radius and time (all nondimensional). The inner boundary moves with the flashing front. The radius of the inclusion is now $1 \mathrm{~cm}$. Other parameters are as in Table 1.

and the constants are determined by the boundary conditions at $r=\varepsilon$ (where the flux value is $-E$ ) and at $r=1$ (where $p=0$ ), so that the steady solution is

$p^{2}=\frac{2 E \varepsilon^{2}}{D}\left(\frac{1}{r}-1\right)$

The value of pressure at $r=\varepsilon$ is then given by

$p(\varepsilon)^{2}=F(1-\varepsilon)$,

where

$F=\frac{2 E \varepsilon}{D}$

This serves as a formula for the maximum pressure that is rapidly approached at the surface of the inclusion in this model. If it exceeds 1 in value, fragmentation is predicted.

\section{Fragmentation criterion}

The crucial combination of parameters in the maximum pressure is $F=\frac{2 E \varepsilon}{D}=\frac{7 R T_{m} K\left(T_{m}-T_{0}\right) \mu_{v}}{M p_{c}^{2} h_{v l} k}$

which is independent of $R_{1}$ and $R_{2}$, so that for $\varepsilon \ll 1$,the maximum pressure is approximated by $p(\varepsilon)=\sqrt{F}$ and this is independent of $R_{1}$ and $R_{2}$. This is consistent with our above observation, that increasing $R_{1}$ from $1 \mathrm{~mm}$ to $1 \mathrm{~cm}$ has little effect on maximum pressure.

However, for larger values of $R_{1}$, the more general formula

$p(\varepsilon)=\sqrt{F(1-\varepsilon)}$

is required, that is,

$p(\varepsilon)=\sqrt{\frac{7 R T_{m} K\left(T_{m}-T_{0}\right) \mu_{v}}{M p_{c}^{2} h_{\mathrm{vl}} k}\left(\frac{R_{2}-R_{1}}{R_{2}}\right)}$

and pressure does vary more significantly with the ratio $\varepsilon=R_{1} / R_{2}$ when it is of order one.

The nature of the dependence on $R_{1}$ is that the maximum pressure reached decreases as $R_{1}$ increases. This may be thought surprising, if one considers that increased $R_{1}$ means more water to drive steam pressures upwards. But increased $R_{1}$ also means a shorter distance 
$R_{2}-R_{1}$ for steam to travel to escape from the magma, reducing pressure rise.

The fragmentation criterion is that $p(\varepsilon)>1$, that is,

$$
\sqrt{\frac{7 R T_{m} K\left(T_{m}-T_{0}\right) \mu_{v}}{M h_{v l} k}\left(\frac{R_{2}-R_{1}}{R_{2}}\right)}>p_{c} .
$$

The full solution of the diffusion problem does depend on $\varepsilon$, as can be seen by comparing Fig. 6 which has $R_{1}=1 \mathrm{~cm}$ (and $R_{2}=100 \mathrm{~cm}$ ) with Fig. 2 which has $R_{1}=1 \mathrm{~mm}$. Both have much the same values for the maximum pressure at $r=\varepsilon$, but the second figure has almost reached steady state throughout by the time the steam source vanishes at $t=1$.

It is also of interest that the fragmentation criterion does not depend on the porosity $\phi_{2}$ of the hot magma surrounding the inclusion. This may be understood as due to the importance of the steady-state pressure solution, which is independent of diffusivity, together with the fact that the speed of the flashing front depends on the temperature gradient driving it from the hot magma, but only on the porosity of the inclusion which is the source of liquid for flashing.

Note to the lack of dependence on $\phi_{1}$. An increased $\phi_{1}$ value slows the speed of the flashing front but increases the amount flashed. In the dimensional problem (Eqs. (6) and (7)) it can correspondingly be seen that in the source term $\frac{R T_{m} \phi_{1} \rho_{1} \dot{s}}{M \phi_{2}}$ the $\phi_{1}$ terms cancel exactly.

\subsection{Travelling flash front}

The steady-state solution is altered slightly in a quasi-steady manner if we solve for steady $p$ on the moving interval $[s(t), 1]$. This gives

$p^{2}(\varepsilon)=\frac{2 E s^{2}}{D}\left(\frac{1}{s}-1\right)$

and in the limit as $s(t)$ approaches zero,

$p^{2}(\varepsilon) \rightarrow \frac{2 E s}{D} \rightarrow 0$

This is consistent with the behaviour of numerical solutions seen in the previous section, in particular with the pressure behaviour at the moving flash front. The steady solution predicts that as $s$ moves towards zero (at a constant time rate), $p$ varies as $\sqrt{s}$, as can be seen in Figs. 5 and 3 as $t \rightarrow 1$.

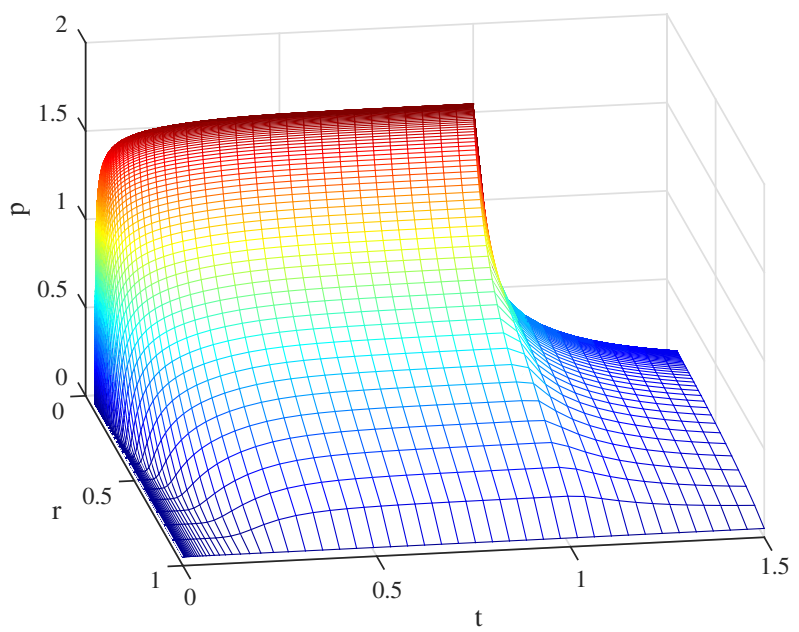

Fig. 6. Numerical solutions to the nondimensional pressure equations, showing pressure versus radius and time (all nondimensional). The flashing front is frozen at its initial location, the surface of the inclusion, which has dimensional radius $1 \mathrm{~cm}$. Other parameters are as in Table 1. a)

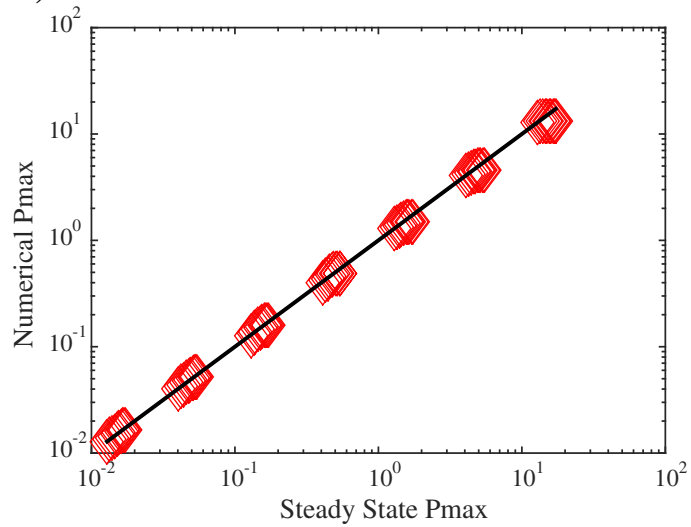

c)

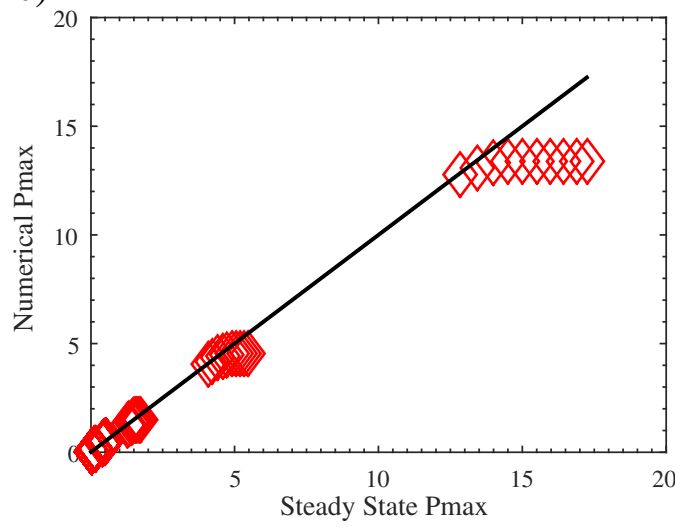

c)

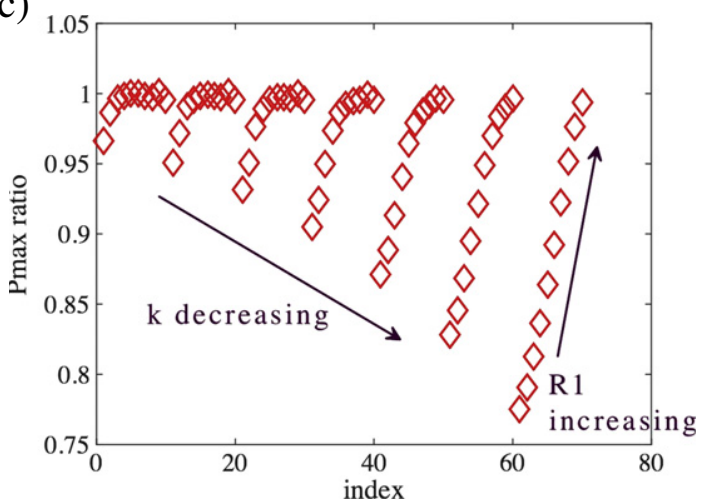

Fig. 7. Comparisons of numerical and theoretical maximum nondimensional pressures at the flashing front in a magma bomb. Numerical results use a point source to give a moving flash front, and cover permeabilities stepping from $10^{-10} \mathrm{~m}^{2}$ to $10^{-16} \mathrm{~m}^{2}$ evenly in log space by dividing by ten, and close groupings of ten values for each permeability of inner radii $R_{1}$ taking values $0.001 \mathrm{~m}, 0.005 \mathrm{~m}$, stepping evenly then to $0.045 \mathrm{~m}$. Symbols indicate numerical values and lines indicate equality when used. Higher maximum pressures correspond to lower permeabilities. Pressure at $r=1$ and initial pressure have been set to zero in these plots. Other parameters are as in Table 1. Trends with decreasing permeability and increasing $R_{1}$ are indicated in the last plot, showing the ratio of numerical to theoretical maximum pressures for each case computed. (a) LogLog plot of numerical vs theoretical maximum pressures (symbols). (b) Linear plot of numerical vs theoretical maximum pressures (symbols). (c) Ratios of numerical to steady-state maximum pressures.

\subsection{Numerical verification}

We tested the fragmentation criterion obtained from the steadystate solution, by comparing it with numerical solutions to the diffusion equation with a travelling flashing front. A number of maximum pressure values were computed at the flashing front, for permeability ranging in powers of ten from $10^{-10} \mathrm{~m}^{2}$ to $10^{-16} \mathrm{~m}^{2}$, and at each 


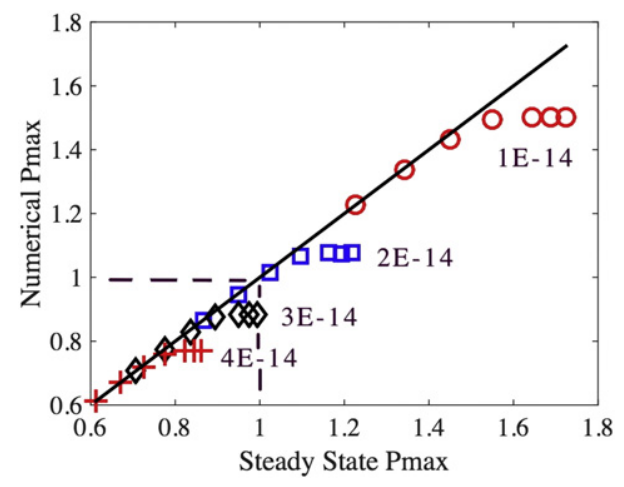

Fig. 8. A close-up view near the critical nondimensional pressure, of a Log-Log plot of numerical vs theoretical maximum pressures (symbols). Permeabilities are $10^{-14} \mathrm{~m}^{2}$ (red circles), $2 \times 10^{-14} \mathrm{~m}^{2}$ (blue squares), $3 \times 10^{-14} \mathrm{~m}^{2}$ (black diamonds), and $4 \times 10^{-14} \mathrm{~m}^{2}$ (red plusses). Each permeability has seven values of inner radii $R_{1}$, $0.001 \mathrm{~m}, 0.005 \mathrm{~m}, 0.01 \mathrm{~m}, 0.02 \mathrm{~m}, 0.03 \mathrm{~m}, 0.04 \mathrm{~m}$, and $0.05 \mathrm{~m}$. As inner radii increase at a fixed permeability, the steady state value of maximum pressure decreases. The critical value of pressure is indicated by the dashed lines. Other parameters are as in Table 1. (For interpretation of the references to colour in this figure legend, the reader is referred to the web version of this article.)

permeability using ten values of $R_{1}$ from the list $0.001,0.005$, then stepping to 0.045 with step-size 0.005 . These computed maximum pressures are compared with the theoretical steady state formula in Fig. 7, where it can be seen that the steady-state approximate formula gives a good match on the log-log plot over a wide range of pressure scales. The linear plots show that the more accurate numerical values of the maximum pressure can be as low as $75 \%$ of those predicted by the formula, and that the better approximations correspond to larger inclusions and to larger permeabilities. That is, for small permeability and small inclusions, the steady-state formula predicts maximum pressures that are higher than observed in numerical simulations. For all of these plots, the initial and boundary pressures have been set to zero.

It is also clear from Fig. 7(a) that it is at a permeability between $10^{-13}$ and $10^{-14} \mathrm{~m}^{2}$ that maximum pressure crosses the critical value of one, irrespective of the value of $R_{1}$.

A comparison of numerical and theoretical maximum pressures over a permeability range that is narrowed to the range $10^{-14} \mathrm{~m}^{2}$ to $2 \times 10^{-15} \mathrm{~m}^{2}$ appears in Fig. 8. The critical value of maximum pressure appears to be reached at permeabilities near $2 \times 10^{-14} \mathrm{~m}^{2}$, depending on $R_{1}$. Rearranging Eq. (20) gives for small $R_{1}$ the critical value of permeability for rupture,

$k_{c}=\frac{7 R T_{m} K\left(T_{m}-T_{0}\right) \mu_{v}}{M h_{v l} p_{c}^{2}} \approx 3 \times 10^{-14} \mathrm{~m}^{2}$.

which is close to our more careful result from numerical simulations.

\section{Steam flow times}

Given the observations of plumes of steam trailing behind Surtseyan bombs, it is of interest to compute the time needed before steam begins to flow through the outer surface of a bomb, and the time period before steam flow is almost exhausted.

The rate of flow of steam $\mathrm{Q}\left(\mathrm{kg} \mathrm{s}^{-1}\right)$ out of the sphere $r=R_{2}$ is given by Darcy's law as

$Q=-4 \pi R_{2}^{2}\left(\frac{k \rho_{v}}{\mu_{v}} \nabla p\right)$

and using the ideal gas law this becomes, in dimensional terms,

$Q=-\frac{4 \pi R_{2}^{2} k M}{\mu_{v} R T_{m}}\left(p \frac{\partial p}{\partial r}\right)$
If we non-dimensionalise as above, this becomes

$Q=-\frac{4 \pi R_{2} k M p_{c}^{2}}{\mu_{v} R T_{m}}\left(\tilde{p} \frac{\partial \tilde{p}}{\partial \tilde{r}}\right)$

Pressure and its derivative are to be evaluated at the outer surface, $\tilde{r}$ $=1$. It is clear that pressure must not be zero here, if $Q$ is to be nonzero, so this section uses $p=p_{a}$ at the outer surface. We calculate $Q(t)$ using the numerical solutions obtained on the moving nondimensional domain $\left[s(t) / R_{2}, 1\right]$, for a variety of parameter values.

Solutions to the diffusion equation have the theoretical property that steam immediately begins to flow out of the bomb, but at infinitesimally small rates initially. The more useful theoretical result is the time at which significant and observable flow rates of steam begin, and the time at which they end. Hence we calculate times for $10 \%, 50 \%$ and $90 \%$ of the included water to escape the bomb.

We have written our pressure diffusion equation in a form that is semi-linear in $p^{2}$ (see Eq. (18)) which has a diffusivity $D p$ that depends on $p$. We use an average value $\bar{p}$ for $p$ in the diffusivity that is based on the steady-state solution. We compute $\bar{p}$ using Eq. (19) for $p^{2}$, and we take the integral average:

$$
\begin{aligned}
\bar{p}=\int_{\varepsilon}^{1} p d r /(1-\varepsilon) & =\sqrt{\frac{2 E \varepsilon^{2}}{D(1-\varepsilon)^{2}}} \int_{\varepsilon}^{1} \sqrt{\frac{1-r}{r}} d r \\
& =\sqrt{\frac{E \varepsilon^{2}}{2 D(1-\varepsilon)^{2}}}\left(2 \sqrt{r-r^{2}}+\sin ^{-1}(2 r-1)\right)_{\varepsilon}^{1} \\
& =\sqrt{\frac{E \varepsilon^{2}}{2 D(1-\varepsilon)^{2}}}\left(\frac{\pi}{2}-2 \sqrt{\varepsilon-\varepsilon^{2}}-\sin ^{-1}(2 \varepsilon-1)\right) .
\end{aligned}
$$

This becomes, for small $\varepsilon$,

$$
\bar{p} \approx \pi \sqrt{\frac{E \varepsilon^{2}}{2 D(1-\varepsilon)^{2}}}
$$

A dimensionless timescale is then given for the lengthscale $1-\varepsilon$ as $\tau=\frac{(1-\varepsilon)^{2}}{D \bar{p}}=\frac{(1-\varepsilon)^{3}}{\varepsilon \pi} \sqrt{\frac{2}{D E}}$.

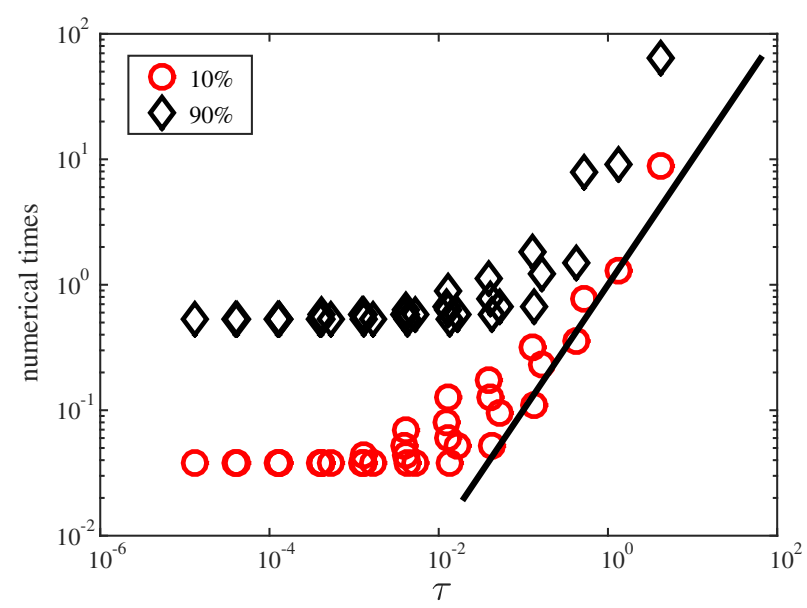

Fig. 9. Plot of the dimensionless times for $10 \%$ (red circles) and $90 \%$ (black diamonds) of the inclusion vapour to flow out of the bomb, versus the theoretical dimensionless timescale $\tau$. The solid line shows where the times would be equal to $\tau$. The cases included here have seven values of permeability stepping from $10^{-10} \mathrm{~m}^{2}$ to $10^{-16} \mathrm{~m}^{2}$ by dividing by ten. For each permeability setting, there are five values of inner radii $R_{1}$, taking values evenly spaced from $0.01 \mathrm{~m}$ to $0.05 \mathrm{~m}$. Other parameter values are as in Table 1. (For interpretation of the references to colour in this figure legend, the reader is referred to the web version of this article.) 
Numerical results were used to find the dimensionless times when $10 \%$ and $90 \%$ of the total amount of water has flowed out of the bomb in vapour form. These are compared with $\tau$ values in Fig. 9, to determine whether $\tau$ is a good measure of steam escape times.

Referring to Fig. 9, it can be seen that at smaller values of $\tau$, the timescale that controls steam release is the time to flash all of the liquid to vapour, a dimensionless time of one. Hence the dimensional timescale for these cases is given by $t_{0}$. This is independent of $\tau$, which is a timescale for diffusion through the magma, not a timescale for flashing. This corresponds to cases where the diffusivity $D$ is so large that vapour flows relatively quickly from the flashing front to the outer surface of the enclosing magma, and the time-limiting factor is the time to flash the liquid to vapour.

At larger values of $\tau$ there is a range of numerical times for a given value of $\tau$, and the trend of these numerical values is the same as that for $\tau$. The value of $\tau$ is seen to provide an approximate lower limit on the $10 \%$ escape times, and about ten times the $\tau$ value provides an estimate of the time for $90 \%$ of the vapour to escape. The errors in these estimates are due largely to approximating the average pressure value using the steady-state solution, but also to the simplification used that $\varepsilon$ is small.

Converting $\tau$ to a predicted timescale $\tau_{s}$ for the time in seconds for most of the water to escape gives

$\tau_{s}=\frac{(1-\varepsilon)^{3}}{\varepsilon \pi} \sqrt{\frac{2}{D E}} t_{0}=\frac{\phi_{2}}{\pi}\left(\frac{R_{2}-R_{1}}{\sqrt{R_{1} R_{2}}}\right) \sqrt{\frac{0.6 \mu_{v} M h_{v l}}{K k R T_{m}\left(T_{m}-T_{0}\right)}}$.

a)

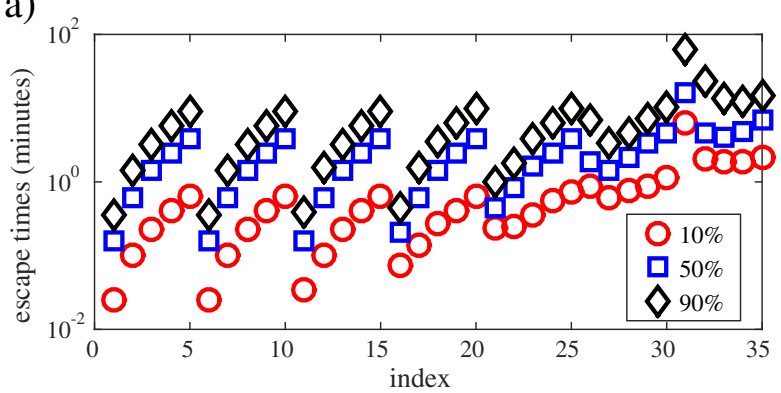

b)

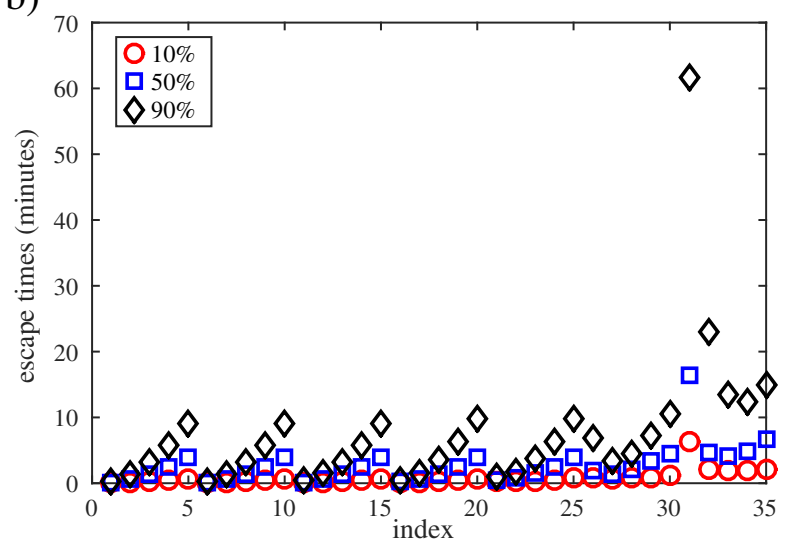

Fig. 10. Plots of the times in minutes for $10 \%, 50 \%$ and $90 \%$ of the inclusion vapour to flow out of the bomb, versus an index for the case run. The upper plot is log-linear, and the lower plot is linear. The cases included here have seven values of permeability stepping from $10^{-10} \mathrm{~m}^{2}$ (index 1-5) to $10^{-16} \mathrm{~m}^{2}$ (index 31-35) by dividing by ten. For each permeability setting, there are five values of inner radii $R_{1}$ increasing from left to right, taking values evenly spaced from $0.01 \mathrm{~m}$ to $0.05 \mathrm{~m}$. Other parameter values are as in Table 1.

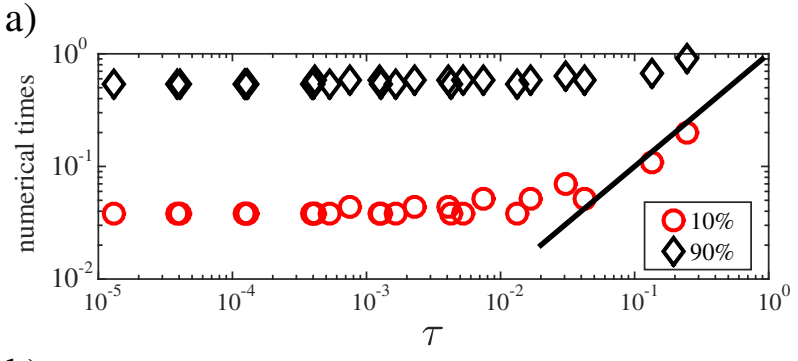

b)

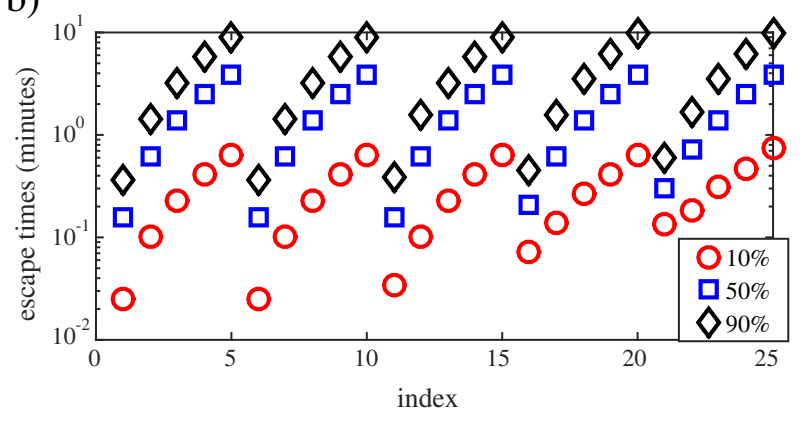

Fig. 11. The cases included here are restricted to bombs which remain intact. There are five values of permeability stepping from $10^{-10} \mathrm{~m}^{2}$ to $10^{-13} \mathrm{~m}^{2}$ by dividing by ten, then a fifth value of $3 \times 10^{-14} \mathrm{~m}^{2}$. For each permeability setting, there are five values of inner radii $R_{1}$, taking values evenly spaced from $0.01 \mathrm{~m}$ to $0.05 \mathrm{~m}$. Other parameter values are as in Table 1. (a) Plot of the dimensionless times for $10 \%$ (red circles) and $90 \%$ (black diamonds) of the inclusion vapour to flow out of the bomb, versus the theoretical dimensionless timescale $\tau$, for bombs that are expected to remain intact. The solid line shows where the times would be equal to $\tau$. (b) Plot of the times in minutes for steam to escape, for bombs that are expected to remain intact. (For interpretation of the references to colour in this figure legend, the reader is referred to the web version of this article.)

Another view of the numerically computed escape times is provided by Fig. 10. It can be seen that that for the higher permeability cases controlled by the time for the inclusion liquid to flash to vapour, escape times have a wide range. Initial appearance of steam ranges from about two seconds for the smallest inclusions in very permeable bombs, to about $50 \mathrm{~s}$ in tight bombs. Most steam has exited a bomb at times ranging from $20 \mathrm{~s}$ for very permeable bombs to forty minutes for tight bombs assuming they have not ruptured. Generally, escape times increase as the square of the radius $R_{1}$ due to the dependence of the timescale $t_{0}$ on $R_{1}^{2}$.

In order to clarify when the flash time dominates, and when the time for vapour to diffuse through the surrounding bomb controls steam release times, plots which are restricted to permeabilities higher than the critical fragmentation value $k=3 \times 10^{-14} \mathrm{~m}^{2}$ for bombs of strength $2 \mathrm{MPa}$ are presented in Fig. 11.

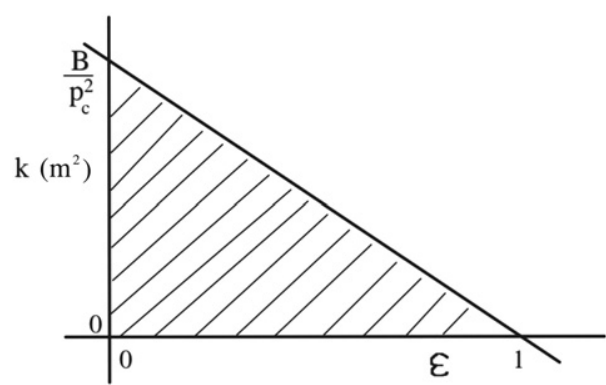

Fig. 12. Region in which fragmentation of a Surtseyan bomb is predicted, in terms of permeability $k$ vs relative inclusion size $\varepsilon=R_{1} / R_{2}$. 


\section{Conclusions}

A nonlinear pressure diffusion equation has been derived that describes anticipated pressure increases due to flashing of a liquid inclusion at the centre of a Surtseyan magma bomb. Numerical solutions and some analysis indicate that, for a range of parameter values, the steady-state solution provides useful information about the maximum pressure difference generated by boiling.

Pressures inside the bomb are highest at the flashing front and decrease with distance from it. They increase rapidly with time at the flashing front. Pressures reach their maximum value at times less than the time to boil all of the inclusion liquid provided that the inclusion has a radius that is less than half of the bomb radius. Rupture is predicted for magma permeabilities less than about $10^{-14} \mathrm{~m}^{2}$, but this depends also on effective tensile strength and other parameters that are quantified in the rupture criterion

$$
\sqrt{\frac{7 R T_{m} K\left(T_{m}-T_{0}\right) \mu_{v}}{M h_{v l} k}\left(\frac{R_{2}-R_{1}}{R_{2}}\right)}>p_{c} .
$$

Noting that the two parameters whose variation from sample to sample might be of most interest are permeability $k$ and the size ratio $\varepsilon=R_{1} / R_{2}$, this criterion may be written in the form

$k<\frac{B}{p_{c}^{2}}(1-\varepsilon)$

where

$B=\frac{7 R T_{m} K\left(T_{m}-T_{0}\right) \mu_{v}}{M h_{v l}}$

This inequality graphs as the region below a straight line as illustrated in Fig. 12.

The graph has slope $-B / p_{c}^{2}$ and intercept $B / p_{c}^{2}$. Using parameter values $T_{m}=1300 \mathrm{~K}, T_{0}=300 \mathrm{~K}$, and $p_{c}=2 \mathrm{MPa}$ gives

$\frac{B}{p_{c}^{2}} \approx 3 \times 10^{-14} \mathrm{~m}^{2}$.

The value $3 \times 10^{-14} \mathrm{~m}^{2}$ gives a lower bound for permeability when the inclusion is negligibly small and bombs are to remain intact. Below this value fragmentation is in general predicted by the mathematical model. Modifying this for larger values of $R_{1}$ is easy using Fig. 12 or Eq. (21).

It is notable that the critical value of permeability given by the steady-state criterion (20) and the consequent approximate value obtained in the previous section of $k=3 \times 10^{-14} \mathrm{~m}^{2}$ for bomb fragmentation is less than the permeabilities of clasts measured by Schipper et al. (Schipper et al., 2013), $10^{-10} \mathrm{~m}^{2}$ to $10^{-13} \mathrm{~m}^{2}$. This measured range of permeabilities of intact bombs is consistent with our theoretical critical value, as the measured values are above the critical value for fragmentation.

We have assumed an effective tensile strength $p_{c}=2 \mathrm{MPa}$. A reduction of this by a factor of three would increase the critical permeability to the value $10^{-13} \mathrm{~m}^{2}$, close to the minimum values measured by Schipper et al. (Schipper et al., 2013).

Steam escape times are found to vary from two seconds to $40 \mathrm{~min}$. They fall into two categories, one controlled by the relatively short time required to flash the entrained liquid water, and the other controlled by the relatively long time required for steam to flow through the vesicular magma bomb. The flashing-controlled cases have initial steam flows observable after about $2 \mathrm{~s}$, and exhaustion of steam venting after about ten minutes. Reference to Fig. 11 makes it clear that these cases also correspond mostly to those bombs which are not expected to fragment if $p_{c}=2 \mathrm{MPa}$, with bombs near fragmentation beginning to show some correlation with the theoretical dimensionless time $\tau$. Other less permeable cases with some distance for the vapour to travel can take up to 40 min to near exhaustion, and the initial appearance of vapour from these bombs may also be significantly delayed, with times ranging from one minute to $20 \mathrm{~min}$. However, these bombs are also the ones with low enough permeabilities that they should fragment, unless the bomb material is stronger than $2 \mathrm{MPa}$.

The timescale $\tau_{s}$ provides a useful indicator of how steam escape times depend on magma properties, but only for bombs with such a small permeability that they are predicted to fragment. For bombs that our model predicts will remain intact, with $k \geq 3 \times 10^{-14} \mathrm{~m}^{2}$, the timescale $t_{0}$ is the relevant one for steam escape times, and this is controlled by the time required to flash the inclusion to steam, not the negligibly small time for the steam to escape from the bomb.

\section{Acknowledgements}

Mark McGuinness acknowledges financial support from Science Foundation Ireland Grant SFI/12/IA/1683.

\section{Appendix A. Thermal lengthscale}

An estimate is required for the thermal lengthscale $R_{T}$, since we approximate the temperature gradient in the magma by $\left(T_{m}-T\right) / R_{T}$. We approximate this lengthscale by estimating how much heat is required to vaporise the liquid in the inclusion, initially at $293 \mathrm{~K}$ and vaporised at $373 \mathrm{~K}$. The heat required to raise the temperature of liquid by $80 \mathrm{~K}$ in the slurry ball with porosity $\phi_{1}$, density $\rho_{l}=1000$, and specific heat $c_{p}^{l}=$ $4184 \mathrm{~J} \mathrm{~kg}^{-1} \mathrm{~K}^{-1}$ is

$Q_{1}=\frac{4}{3} \pi R_{1}^{3} \phi_{1} \rho_{l} c_{p}^{l} 80 \approx 1.4 \times 10^{6} \phi_{1} \rho_{l} R_{1}^{3}$.

A similar calculation for the heat $Q_{2}$ required to raise the temperature of the solid component of the inclusion gives $Q_{2} \approx 0.5 Q_{1}\left(1-\phi_{1}\right) /$ $\phi_{1}$. The heat required to vaporise at $373 \mathrm{~K}$ is greater:

$Q_{3}=\frac{4}{3} \pi R_{1}^{3} h_{v l} \phi_{1} \rho_{l} \approx 9.5 \times 10^{6} \phi_{1} \rho_{l} R_{1}^{3}$

where $h_{v l} \approx 2260 \mathrm{~kJ} \mathrm{~kg}^{-1}$ is the specific heat of vaporisation.

Hence an effective value for $c_{p}$ that applies up to the point that all of the liquid in the inclusion is vaporised can be estimated as about $Q_{3} / Q_{1}$ times the value 4184 used above, that is, $c_{p} \approx 3 \times 10^{4} \mathrm{~J} \mathrm{~kg}^{-1} \mathrm{~K}^{-1}$.

The volume of magma in a shell of radius $R_{1}+R_{T}$ about the inclusion is

$V_{T}=\frac{4}{3} \pi \phi_{2}\left[\left(R_{1}+R_{T}\right)^{3}-R_{1}^{3}\right]$

and the sensible heat due to a change of temperature of $900 \mathrm{~K}$ in this is set equal to $Q_{1}+Q_{2}+Q_{3}$ to find $R_{T}$ :

$900 c_{m} \phi_{2} \rho_{m} V_{T}=Q_{1}+Q_{2}+Q_{3}$

where the thermal capacity $c_{m}=840 \mathrm{~J} \mathrm{~kg}^{-1} \mathrm{~K}^{-1}$, magma density $\rho_{m}=$ $2800 \mathrm{~kg} \mathrm{~m}^{-3}$, and magma porosity is $\phi_{2}$. This gives

$\left(R_{1}+R_{T}\right)^{3} \approx\left[1+1.2 \frac{\phi_{1}}{\phi_{2}}+0.6\left(\frac{1-\phi_{1}}{\phi_{2}}\right)\right] R_{1}^{3}$

Using equal porosities in magma and inclusion with values of 0.4 then gives the estimate for thermal lengthscale

$R_{T} \approx 0.3 R_{1}$ 


\section{References}

Crank, J., 1974. The Mathematics of Diffusion. second ed. Clarendon Press, Oxford. Goodman, R., 1989. Introduction to Rock Mechanics. Wiley, NY.

Grant, M.A., 1978. Two-phase linear geothermal pressure transients: a comparison with single-phase transients. N. Z. J. Sci. 21, 355-364

Kokelaar, B., 1983. The mechanism of Surtseyan volcanism. J. Geol. Soc. 140, 939-944.

Kokelaar, P., 1986. Magma-water interactions in subaqueous and emergent basaltic volcanism. Bull. Volcanol. 48, 275-289.

Koyaguchi, T., Scheu, B., Mitani, N., Melnik, O., 2008. A fragmentation criterion for highly viscous bubbly magmas estimated from shock tube experiments. J. Volcanol. Geotherm. Res. 178, 58-71.

Moench, A.F., 1979. Radial Steam Flow in Two-phase Geothermal Reservoirs Comparison of Analytical and Finite-difference Solutions for Transient Pressure Drawdown, Paper SPE 7959 Presented at the 1979 California Regional Meeting of the Soc. of Petroleum Engineers of AIME Held in Ventura, California April. pp. 1-5.

Moench, A.F., Atkinson, P., 1977. Transient Pressure Analysis in Geothermal Steam Reservoirs With an Immobile Vaporizing Liquid Phase - Summary Report, Proceedings of the Third Workshop on Geothermal Reservoir Engineering. Stanford University, California, pp. 1-5 Dec. 14-16.

Mueller, S., Scheu, B., Spieler, O., Dingwell, D., 2008. Permeability control on magma fragmentation. Geology 36, 399-402.
Murtagh, R.M., White, J.D., 2013. Pyroclast characteristics of a subaqueous to emergent Surtseyan eruption, black point volcano, California. J. Volcanol. Geotherm. Res. 267, 75-91.

Schipper, C.I., White, J.D., Magma-slurry interaction in surtseyan eruptions, Geology, Data Repository item 2016057, http://dx.doi.org/10.1130/G37480.1.

Schipper, C., Burgisser, A., White, J., 2013. Magma permeability and magma-slurry mingling during the 1963-67 eruption of Surtsey, Iceland. Japan Geoscience Union Meeting, Chiba, Japan vol. 02519 (SVC50-P09).

Spieler, O., Kennedy, B., Kueppers, U., Dingwell, D., Scheu, B., Taddeucci, J., 2004. The fragmentation threshold of pyroclastic rocks. Earth Planet. Sci. Lett. 226, 139-148.

Thorarinsson, S., 1965. The surtsey eruption: course of events and the development of the new island. Surtsey Res. Prog. Rep. 1, 50-55.

Thorarinsson, S., 1967a. The Surtsey eruption: course of events during the year 1966. Surtsey Res. Prog. Rep. 3, 84-92.

Thorarinsson, S., 1967b. Surtsey: The New Island in the North Atlantic, Viking, New York. Thorarinsson, S., 1968. The Surtsey eruption: course of events during the year 1967. Surtsey Res. Prog. Rep. 4, 143-148.

Thorarinsson, S., Einarsson, T., Sigvaldason, G., Elisson, G., 1964. The submarine eruption off the Vestmann islands 1963-64: a preliminary report. Bull. Volcanol. 27, 435-445.

White, J.D., Houghton, B., 2000. Surtseyan and related phreatomagmatic eruptions. In: Sigurdsson, H., Houghton, B.F., McNutt, S.R., Rymer, H., Stix, J. (Eds.), Encyclopedia of Volcanoes. Academic Press, New York, pp. 495-511. 